\title{
The inverted pattern of circulating miR-221-3p and miR-222-3p associated with isolated low HDL-C phenotype
}

\author{
Yuntao Zhou*, Mengdi Liu, Jinrong Li, Bing Wu, Wei Tian*, Lu Shi, Jing Zhang and Zening Sun
}

\begin{abstract}
Background: We investigated the baseline characterization of cardiovascular disease (CVD)-derived circulating miR-221-3p/222-3p in isolated low HDL-C phenotype (ILHP) to enhance our understanding on their molecular pathological pattern prior to disease onset.
\end{abstract}

Methods: We screened 174 asymptomatic subjects with isolated low HDL-C phenotype $(n=88)$ and normal lipid phenotype $(n=86)$, and detected circulating levels of CVD-derived circulating miR-221-3p/222-3p using TaqMan miRNA Real-time PCR detection system.

Results: We found the inverted pattern of decreased circulating miR-221-3p $(0.415[0.249,1.004]$ vs $0.658[0.347,1.534]$, $p=0.002)$ versus increased miR-222-3p levels $(0.379[0.101,0.701]$ vs $0.156[0.043,0.407], p<0.001)$ in ILHP. The baseline levels of circulating miR-221-3p and miR-222-3p are correlated with serum HDL-C levels (miR-221-3p: $r=0.306$, $p<0.001$; miR-222-3p: $r=-0.201, p=0.008$ ). Gender-based analysis showed female-specific elevation of circulating miR-221-3p in asymptomatic individual. Multiple logistic regression analysis showed that circulating miR-222-3p is robustly independent factor (adjusted $\mathrm{OR}=8.42,95 \% \mathrm{Cl}: 2.53-27.98, p<0.001$ ) and significantly improved the performance of the predictive clinical model distinguished ILHP from normal lipid phenotype (AUC: $0.816,95 \% \mathrm{Cl}(0.754,0.879)$ vs AUC: $0.771,95 \% \mathrm{Cl}(0.702,0.840) ; Z=2.169, p=0.030)$. Moreover, the increased original Ct ratio of miR-221-3p to miR-222-3p in male ILHP $(1.003[0.927,1.063]$ vs $0.927[0.858,0.967], p<0.001)$ significantly enhanced the ability to classify male ILHP compared with the male predictive clinical model (AUC: 0.851 , $95 \% \mathrm{Cl}(0.770,0.933)$ vs AUC: $0.759,95 \% \mathrm{Cl}(0.659,0.859) ; Z=2.474, p<0.05)$.

Conclusions: The inverted pattern of circulating miR-221-3p and miR-222-3p are potentially clinically actionable signature for molecular pathology in isolated low HDL-C phenotype.

Keywords: Isolated risk phenotype, Circulating microRNAs, Cardiovascular disease, Clinical translation

\section{Background}

MicroRNAs (miRNAs) are a class of endogenous small non-coding regulatory RNAs that play important roles in the regulation of various physiological and pathological processes [1]. Besides the intracellular biological functions, miRNAs, which can be exported or released into the extracellular space in multiple stable forms, have been proposed as innovative biomarker surrogates for human diseases [2]. Over the past few years, altered

\footnotetext{
* Correspondence: yuntaozhou@hotmail.com; tianwei0@hotmail.com Tangshan Key Laboratory of Clinical Molecular Diagnosis and Treatment, Tangshan Gongren Hospital, No. 27 Wenhua Road, Tangshan, Hebei 063000, People's Republic of China
}

levels of miRNAs, involved in lipid and cholesterol metabolism as well as cardiovascular development and remodelling [3, 4], have been reported in plasma or serum from patients with atherosclerotic cardiovascular diseases such as acute myocardial infarction (AMI) [5] and coronary artery disease (CHD) [6]. In contrast to most candidates, cardio-specific miRNAs exhibit the robust performance in the diagnosis, prediction and prognosis of developing acute coronary syndrome (ACS) patients [7, 8]. However, clinical uses for most of circulating miRNA candidates altered in ACS patients have been not well definitely elucidated yet $[9,10]$. Our previous studies showed that miR-28-5p involved in 
ERK2-mediated ABCA1 up-regulation did not exhibit the strong association between its increased plasma levels and higher HDL-C levels in UAP patients as expected [11]. This might be attributed to the complexity and uncertainty of established ACS patients besides its relative low levels in plasma. Some studies suggest that the changes of circulating miRNAs in cardiovascular diseases could be found easier than their intrinsic nature or interactive relationships with known or unknown factors behind combinational confounding variables [12-15]. Therefore, we are intending to investigate circulating miRNAs in asymptomatic individuals with isolated cardiovascular risk phenotype to minimize various potential confindings effects and understand their baselines in the isolated risk population.

Isolated low levels of high-density lipoprotein cholesterol (HDL-C) named isolated low HDL-C phenotype is described as a condition that coexists low HDL-C level in combination with normal LDL-C and triglyceride (TG) levels [16]. It is the most common lipid abnormality among different ethnic populations associated with increased ACS risk, especially for the Asian population $[16,17]$. Individuals with isolated low HDL-C phenotype are predisposed to exhibit qualitative and quantitative traits including genetic variations [18], endothelial dysfunction [19], proinflammatory and oxidative phenotype [20], the specific composition and lipid spatial distribution of HDL particles [21], and low cholesterol efflux [22], as well as enhanced lipid peroxidation and platelet activation [23]. These changes are also observed frequently in patients with atherosclerotic diseases. However, the pattern of cardiovascular disease-related circulating miRNA candidates in isolated low HDL-C phenotype remain unknown. Decipering cardiovascular disease-derived circulating miRNA candidates in isolated low HDL-C phenotype would be useful to understand isolated phenotype-associated pathological features at circulating miRNA level.

An another key issue is how to select circulating miRNA candidates for isolated low HDL-C phenoytype. It is universally acceptable that either gender or genetic factors have major effects on HDL-C level. Based on dominant effect of gender factor on it, we firstly filtered the $\mathrm{X}$-chromosome-located miRNA candidates and found the significantly increased levels of single-primary-prescursor-derived miR-221/222 mature transcripts in plasma from UAP patients (http://www.ncbi.nlm.nih.gov/geo/, accession number: GSE94605). The transcription of miR-221/222 has been reported to be controlled by estrogen receptor alpha-mediated signaling pathway [24] and ApoE-containing HDL particles [25]. Moreover, miR-222 has been reported to be transported into circulation via HDL particles [26]. Taken together these evidence, we investigated plasma miR-221/222 mature transcripts in isolated low HDL-C phenotype, and analyzed their relationships with isolated low HDL-C phenotype, gender factor and ApoE genotyping.

\section{Methods \\ Study population}

One hundred seventy-four asymptomatic subjects with isolated low HDL-C phenotype or normal lipid profiles were enrolled from Medical Health Check-up Department in Tangshan Gongren Hospital between August 2014 and November 2015. All subjects were screened according to the following criteria: 1 ) subjects who are less than 55 years of age; 2) subjects who were excluded if they had obesity, metabolic syndrome, diabetes, hypertension, kidney disease, and cardiovascular disease and recent medication history. Laboratory evalution included fasting Glucose, serum lipids, hepatic injury indexes. This study was approved by the Ethnics Committee of Tangshan Gongren Hospital and the written informed consent was obtained from all subjects.

\section{Blood samples collection and handling}

Peripheral blood was drawn with EDTA-K2-anticoagulated tube and handled within $2 \mathrm{~h}$. Whole blood samples were centrifuged at 3, $000 \mathrm{~g}$ for $10 \mathrm{~min}$ followed by centrifugation at $12,000 \mathrm{~g}$ for $10 \mathrm{~min}$ to generate platelet- and cell debris-deficient plasma. The deprived plasma samples were immediately stored at $-80^{\circ} \mathrm{C}$.

\section{Isolation and purification of plasma miRNAs}

RNA was isolated from a fixed volume $(500 \mu \mathrm{L})$ of plasma using Ambion mirVana ${ }^{\mathrm{Tm}}$ PARIS RNA isolation kit (Invitrogen) according to the manufacturer's instructions. Extracted RNA were dissolved in a fixed volume $(90 \mu \mathrm{L})$ of elution solution and then stored at $-80^{\circ} \mathrm{C}$.

\section{miRNA quantification with RT-qPCR}

miRNA detection were performed with TaqMan miRNA Reverse Transcription Kit, TaqMan MiRNA Assays and TaqMan Universal PCR Master Mix (Applied Biosystems) on ABI 7500 PCR system according to the manufacturer's protocol and modified protocol. The reaction of miRNA reverse transcription containing $1.2 \mu \mathrm{L} 10 \times$ reverse transcription (RT) buffer, a fixed volume of 8.6 $\mu \mathrm{L}$ RNA elution from the $90-\mu \mathrm{L}$ eluate of RNA isolation as RT template, $0.1 \mu \mathrm{L} 100 \mathrm{mM}$ dNTPs, $0.1 \mu \mathrm{L}$ RNase inhibitor $(20 \mathrm{U} / \mu \mathrm{L}), 1.5 \mu \mathrm{L}$ RT primer, $0.5 \mu \mathrm{L}$ MultiScribe $^{\mathrm{Tw}}$ reverse transcriptase $(50 \mathrm{U} / \mu \mathrm{L})$ were performed following parameters: $16{ }^{\circ} \mathrm{C}$ for $60 \mathrm{~min}, 42{ }^{\circ} \mathrm{C}$ $120 \mathrm{~min}$ and $85^{\circ} \mathrm{C} 5 \mathrm{~min}$. The PCR reaction comprised $6 \mu \mathrm{L}$ RT product, $10 \mu \mathrm{L}$ of $2 \times$ TaqMan Universal PCR Master Mix, $0.5 \mu \mathrm{L}$ TaqMan probe $(2.5 \mu \mathrm{M})$, and $3.5 \mu \mathrm{L}$ DNase/RNase-free $\mathrm{H}_{2} \mathrm{O}$, according to the following parameters: $95{ }^{\circ} \mathrm{C} 10 \mathrm{~min}$ followed by 50 cycles of $95{ }^{\circ} \mathrm{C}$ 
for $15 \mathrm{~s}, 60$ for $1 \mathrm{~min}$. Cycle threshold $(\mathrm{Ct})$ values were determined on ABI 7500 real-time PCR system (Applied Biosystems). Mature transcripts of miR-221/222 in plasma were detected using TaqMan miRNA detection system. miR-191-5p was used as an endogenous reference for miRNA quantification as previously reported [27]. A Ct value $>40$ was defined as undetectable for all miRNAs. Relative expression level was determined with the $\Delta \mathrm{Ct}$ method and reported as $2^{-\Delta \mathrm{Ct}}$.

\section{APOE genotype}

Genomic DNA were extracted from whole blood samples using TIANamp blood DNA kit (TIANGEN Biotech (Beijing) Co., Ltd). The APOE gene has three variant alleles $(\varepsilon 2, \varepsilon 3, \varepsilon 4)$ which generate six different genotypes $(\varepsilon 2 / 2, \varepsilon 2 /$ $3, \varepsilon 2 / 4, \varepsilon 3 / 3, \varepsilon 3 / 4$ and $\varepsilon 4 / \varepsilon 4)$. APOE allele carriers were defined as follows: 1) the E2 carrier includes those subjects with the $\varepsilon 2 / \varepsilon 2$, $\varepsilon 2 / \varepsilon 3$ or $\varepsilon 2 / \varepsilon 4$ genotype; 2 ) the E3 carrier includes those with the $\varepsilon 3 / \varepsilon 3$ genotype; and 3 ) the E4 carrier includes those with the $\varepsilon 3 / \varepsilon 4$ or $\varepsilon 4 / \varepsilon 4$ genotype. E2 has been shown to be associated with decreased LDL-C levels, whereas E4 is associated with increased CVD risk [28]. $A P O E$ genotyping analysis was performed using $\mathrm{ABI}$ $\mathrm{PRISM}^{\circ} \mathrm{SNaPshot}^{\mathrm{Tu}}$ M $\mu$ ltiplex Kit (Applied Biosystems) on ABI 9700 PCR system and 3730XL DNA analyzer.

\section{Statistical analysis}

The statistic analyses were performed using GraphPAD Prism 5.0 and $\mathrm{R}$ bioconductor 3.0.1. Continuous variables were tested for normal distribution with the D'Agostino and Pearson ominbus normality test. Normally distributed continuous variables were presented as mean \pm SD. Two groups were compared using the Student $t$ test and the Mann-Whitney $U$ test, as appropriate. Multiple comparisons were compared using the Kruskal-Wallis test, and the $P$ values were corrected using the Benjamini-Hochberg (BH) method to control the false discovery rate. Spearman rank correlation test was performed to identify the possible relationships between circulating miRNAs and baseline characteristics of clinical laboratory parameters. Receiver operating characteristic (ROC) curves were used to evaluate the performance of univariate or multivariate logistic model. The optimal cut-off point of ROC curve was chosen at which the Youden's index was maximal. Linear or logistic regression analysis was performed to explore the associations among circulating miRNAs, $A P O E$ allele carriers and isolated low HDL-C phenotype, find the optimized combination of predictive variables and build a model of predicted probability for defining isolated low HDL-C phenotype. Variables that were not normally distributed were transformed to their natural logarithm in linear regression analysis. $p<0.05$ was considered to indicate statistical significance.

\section{Results}

Baseline characteristics of participants

One hundred seventy-four subjects (88 for isolated low HDL-C phenotype and 86 for normal lipid phenotype, Additional file 1: Table S1) were screened from approximately more than 400 participants from Healthy Check-up Department during August, 2014 to November, 2015. All subjects enrolled in this study shared common traits including normal non-HDL-C profiles $(\mathrm{TC}<6.00 \mathrm{mmol} / \mathrm{L}, \mathrm{LDL}-\mathrm{C}<3.30 \mathrm{mmol} / \mathrm{L}$ and $\mathrm{TG}<$ $1.70 \mathrm{mmol} / \mathrm{L})$, fasting glucose $(\mathrm{FG}<6.10 \mathrm{momol} / \mathrm{L})$, biochemistry tests of liver injury (ALT and AST $\leq 40$; GGT $\leq 50$ ) and the removal of other known CVD risk factors. Isolated low HDL-C phenotype is defined as subjects with $\mathrm{HDL}-\mathrm{C}$ level $<1.04 \mathrm{mmol} / \mathrm{L}$ in male or $1.16 \mathrm{mmol} / \mathrm{L}$ in female refered to phenotype-associated publication [29], especially for female. As shown in Additional file 1: Table S1, three of 15 non-HDL parameters within normal reference range had significant difference between isolated low HDL-C and normal lipid phenotype $(p<0.001$ for TC and TG; $p=0.032$ for ALT). Gender-based clinical features as shown in Additional file 1: Table S2. Several variables including TC, HDL-C, TG, ALT, AST, GGT, HGB, PLT and RBC had significant difference among gender-based subgroups.

\section{Circulating miR candidates and isolated low HDL-C phenotype}

Relative levels of miR-221-3p and miR-222-3p in plasma from asymptomatic subjects $(n=174)$ with normal lipid phenotype or isolated low HDL phenotype were detected using TaqMan stem-loop miRNA assay system. miR-221-3p and 222-3p as well as an endogenous miRNA, miR-191-5p were clearly detected in all of plasma samples. There were decreased levels of miR-221-3p $(0.415$ [0.243, 1.001] vs 0.658 [0.339, 1.574]; $p=0.002$; Fig. 1a) and increased levels of miR-222-3p (0.379 [0.086, 0.707] vs 0.156 [0.042, 0.409]; $p<0.001$; Fig. 1b) in isolated low HDL phenotype $(n=88)$ in comparison with normal lipid phenotype $(n=86)$. The inverted pattern of miR-221-3p and miR-222-3p in isolated low HDL-C phenotype is similar with that in morbid obesity [30].

In gender-based subgroup analysis, miR-221-3p $\left(x^{2}=\right.$ 28.36, $p<0.001$; Fig. $1 c)$ and miR-222-3p $\left(x^{2}=11.92, p\right.$ $=0.01$; Fig. $1 \mathrm{~d}$ ) were found to be significant difference. As shown in Fig. 1c, circulating miR-221-3p levels were predominantly elevated in female with either isolated low HDL-C phenotype or normal lipid phenotype compared with the male subgroups, which is similar to female-specific elevation in participants with metabolic syndrome [31]. Notably, increased circulating miR221-3p levels were observed between male-categorized subgroups $(0.320$ [0.180, 0.428] vs 0.466 [0.293, 1.315], 

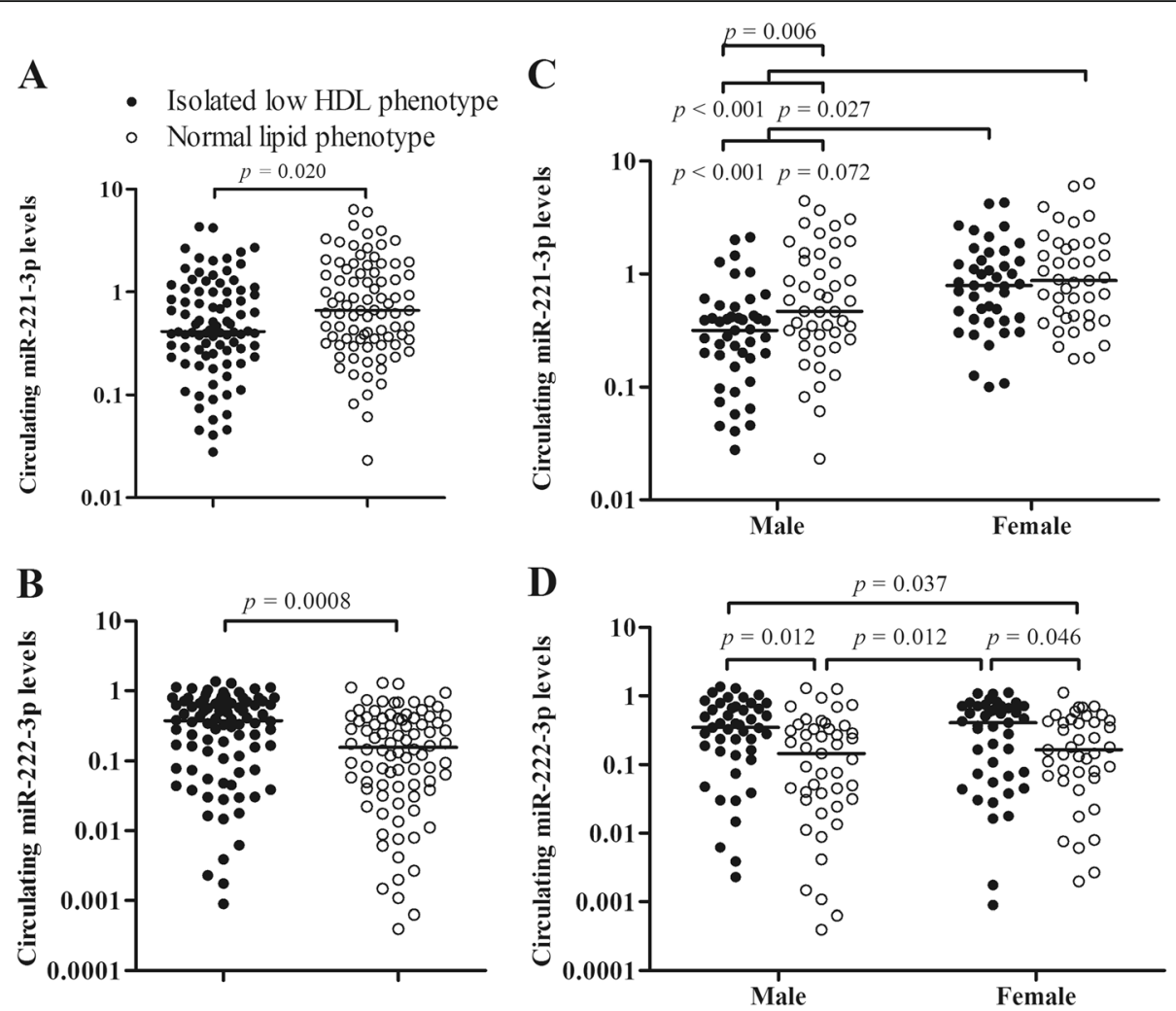

Fig. 1 Circulating miRNAs levels in isolated low HDL-C phenotype. Circulating miR-221-3p levels (a) are increased in isolated low HDL-C phenotype, whereas circulating miR-222-3p levels (b) are decreased in isolated low HDL-C phenotype. Isolated low HDL: Isolated low HDL-C phenotype; Normal: Normal lipid phenotype. miR-221-3p levels (c) are significantly increased in female subjects with both isolated low HDL-C phenotype and normal lipid phenotype in comparison with those of male subgroups. $\mathbf{d}$ Circulating miR-222-3p levels are significantly increased in either male or female isolated low HDL-C phenotype compared with the corresponding normal lipid phenotypes, respectively. The $p$ value is adjusted using the Benjamini-Hochberg (BH) method in multiple comparisons

$p=0.006)$, rather than between female subgroups $(0.790[0.405,1.311]$ vs $0.875[0.428,1.824], p=0.288)$. Nevertheless, the increases of circulating miR-222-3p were predominantly observed in both of intra-gender isolated low HDL subgroups compared with the correponding normal lipid phenotype (male: 0.351 [0.158, 0.663 ] vs 0.147 [0.032, 0.335], $p=0.012$; female: 0.413 $[0.071,0.712]$ vs 0.166 [0.069, 0.432], $p=0.046$; Fig.1d).

\section{The association of baseline levels of clinical laboratory indexes and miR candidates}

The relationships between clinical laboratory indexes and circulating miRNAs levels in asymptomatic subjects $(n=174)$ with both normal lipid phenotype and isolated low HDL-C phenotype were firstly analyzed using Spearman's correlation analysis and linear regression analysis. Serum HDL-C levels were correlated with both of miRNA candidates (miR-221-3p: $r=0.306, p<0.001$, Fig.2a; miR-222-3p: $r=-0.201, p=0.008$, Fig.2b). The correlations with other clinical indexes were shown in Additional file 1: Table S3. Circulating miR-222-3p levels were not correlated with all the other clinical indexes (all $p>0.05$ ), whereas circulating levels of miR-221-3p were correlated with 7 non-HDL-C clinical indexes, respectively $(p<0.05)$. Furthermore, step-wise linear regression analysis showed that plasma miR-222-3p levels were associated with HDL-C levels and absolute count of RBC, whereas plasma miR-221-3p were associated with five clinical laboratory indexes including gender, HDL-C, LDL-C, etc. (Additional file 1: Tables S4 and S5).

Binary logistic regression analyses were further performed to deduce the association between isolated low HDL-C phenotype and the two miRNA candidates. Our results showed that isolated low HDL-C phenotype was strongly associated with increased circulating miR-222-3p levels (adjusted $\mathrm{OR}=8.42$, 95\%CI: 2.53-27.98, $p<0.001$ ), rather than that of miR-221-3p (adjusted OR $=0.67,95 \% \mathrm{CI}$ : $0.45-1.00, p$ $=0.051$; Table 1) after adjustment for baseline characteristics of non-HDL clinical laboratory parameters. These results indicate that circulating miR-222-3p level is an independent factor for isolated low HDL-C phenotype. 

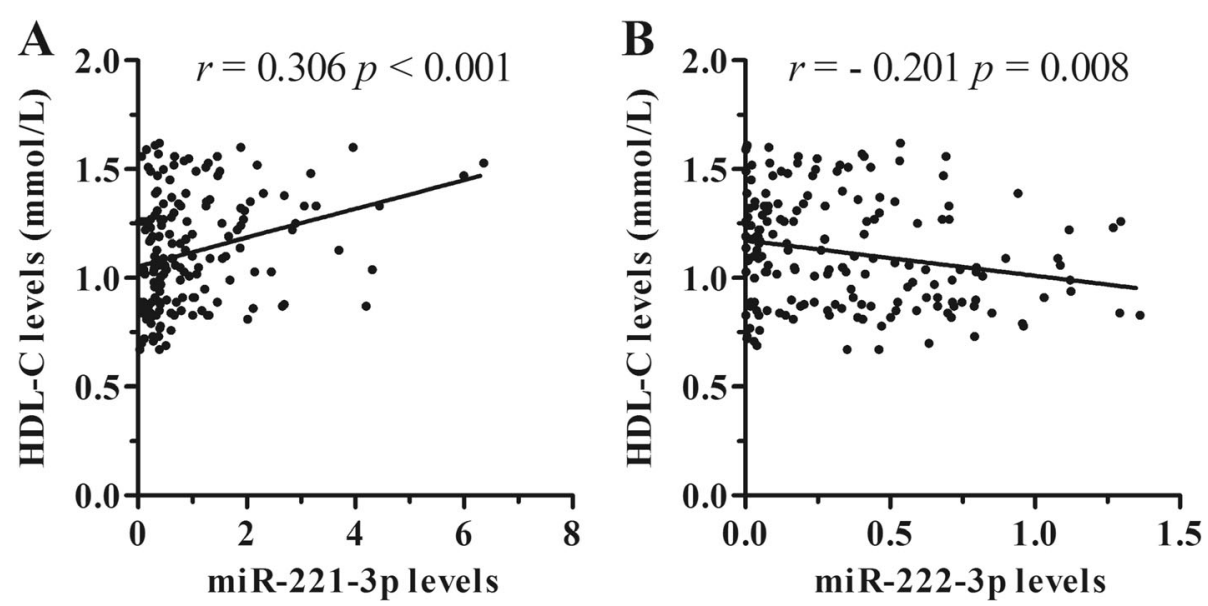

Fig. 2 The correlations between circulating miRNA levels and serum HDL-C levels. Circulating miR-221-3p levels (a) are positively correlated with serum HDL-C levels, whereas circulating miR-222-3p levels $(\mathbf{b})$ are inversely correlated with serum HDL-C levels in asymptomatic subjects ( $n=174)$

Circulating miR-222-3p improve the performance of the predective clinical model for isolated low HDL-C phenotype

Predictive clinical model was firstly estimated using step-wise regression analysis (Additional file 1: Table S6), and then ROCs' performance of circulating miR-222-3p on defining isolated low HDL-C phenotype was analyzed compared with the predictive clinical model. As shown in Table 2, univariate miR-222-3p yielded an AUC of 0.648 (95\% CI, 0.566-0.730; $p<0.001$ ). The AUC performance was significantly improved when adding miR-222-3p to the predictive clinical model (AUC: $0.816,95 \% \mathrm{CI}$ : 0.754, 0.879 vs (AUC: $0.771,95 \% \mathrm{CI}$ : 0.702-0.840; $Z=2.169, p=0.030$ ).

\section{Using the Ct ratio of miR-221-3p and miR-222-3p to define male isolated low HDL-C phenotype}

According to the opposite changes of miR-221-3p and miR-222-3p in isolated low HDL-C phentype, the ratio of miR-221-3p to miR-222-3p original $C t$ value was analyzed. The miR-221-3p/222-3p ratios were significantly increased in isolated low HDL-C phenotype in comparison with normal lipid phenotype $(0.991$ [0.894, 1.044] vs 0.924 [0.855, 0.976], $p<0.001$; Fig.3a). Compared with the corresponding normal phenotypes, male isolated low HDL phenotype exhibited significantly increased ratio (1.003 [0.927, 1.063] vs 0.927 [0.858, 0.967], $p<0.001)$, while female had an increased trendence (0.979 [0.871, 1.009] vs 0.922 [0.846, 0.976], $p=0.062$; Fig.3b).
Correlation analysis showed that the miR-221-3p/ 222-3p ratios were inversely correlated with serum HDL-C levels $(r=-0.333, p<0.001$; Fig. 3c). Moreover, the substitution of the miR-221/222 ratio for circulating miR-221-3p and miR-222-3p levels showed the compromised features with the other clinical indexes (Additional file 1: Table S3).

Multivariate logistic regression analysis showed that the miR-221-3p/miR-222-3p ratio yielded an adjusted OR value of 5617.96 (95\% CI: 59.09-534,118.17, $p<0.001$, Table 1). As shown in Table 2, introducing the miR-221-3p/ miR-222-3p ratio slightly improved the performance of the predictive clinical model on distinguishing isolated low HDL-C phenotype from normal lipid phenotype $(Z=1.972$, $p=0.049)$ although there was no significant difference between the original $\mathrm{Ct}$ ratio and circulating miR-222-3p level $(Z=-0.225, p=0.821)$. In gender-based analysis (Table 3 ), addition of miR-221-3p/miR-222-3p ratio $(Z=2.474, p=$ $0.013)$, instead of miR-222-3p level $(Z=1.844, p=0.065)$, significantly enhanced the performance of the male predictive clinical model, while neither did miR-221-3p/ miR-222-3p ratio nor circulating miR-222-3p level did improve that of the female clinical model (All $p>0.05$ ).

\section{APOE genotype have no effect on circulating miR-221/ 222-3p}

Considering ApoE-mediated inhibitory effect on miR$221 / 222$ under cellular condition as well as the

Table 1 Associations of circulating miRNA indexes with isolated low HDL-C phenotype

\begin{tabular}{|c|c|c|c|c|c|c|}
\hline \multirow[t]{2}{*}{ Models } & \multicolumn{2}{|l|}{ miR-221-3p } & \multicolumn{2}{|l|}{ miR-222-3p } & \multicolumn{2}{|l|}{ miR-221-3p/222-3p Ct ratio } \\
\hline & OR $(95 \% \mathrm{Cl})$ & $p$ value & OR $(95 \% \mathrm{Cl})$ & $p$ value & OR $(95 \% \mathrm{Cl})$ & $p$ value \\
\hline Crude & $0.67(0.49,0.93)$ & 0.018 & $5.45(1.99,14.88)$ & $<0.001$ & $996.49(27.34,36,324.83)$ & $<0.001$ \\
\hline Adjusted, model ${ }^{a}$ & $0.67(0.45,1.00)$ & 0.051 & $8.42(2.53,27.98)$ & $<0.001$ & $5617.96(59.09,534,118.17)$ & $<0.001$ \\
\hline
\end{tabular}

a Model: adjusted for clinical laboratory indexes including LDL-C, TG, ALT, AST, GGT, FG, HGB, PLT, and absolute counts of WBC, RBC, neutrophil and lymphocyte, except for HDL-C. OR odd ratio, Cl confidence interval 
Table 2 The performance of circulating miR-222-3p and miR-221/.222 Ct ratio for predicting isolated low HDL-C phenotype

\begin{tabular}{llllll}
\hline Models & AUC $(95 \% \mathrm{Cl})$ & $p$ value & Cut-off value & Specificity & Sensitivity \\
\hline miR-222-3p & $0.648(0.566,0.730)$ & $<0.001$ & 0.559 & 0.837 & 0.432 \\
The miR-221-3p/miR-222-3p ratio & $0.678(0.598,0.758)$ & $<0.001$ & 0.562 & 0.767 & 0.568 \\
Clinical model & $0.771(0.702,0.840)$ & $<0.001$ & 0.462 & 0.698 & 0.761 \\
Clinical model + miR-222-3p ${ }^{a}$ & $0.816(0.754,0.879)^{b}$ & $<0.001$ & 0.413 & 0.663 & 0.879 \\
Clinical model + miR221-3p/miR-222-3p ratio ${ }^{\text {a }}$ & $0.813(0.751,0.876)^{b}$ & $<0.001$ & 0.423 & 0.686 & 0.818
\end{tabular}

AUC area under the curve. Best threshold of Cut-off value were estimated using youden method. ${ }^{a}$ The AUC, sensitivities and specificities of the combination model of miR-222-3p and clinical model was compared with that of clinical model. ${ }^{b} p<0.05$

evaluation on whether participants were randomly enrolled in this study $[25,28]$, we performed $A P O E$ genotyping and Hardy-Weinberg equilibrium (HWE) testing. Genotypes and allele frequencies of $A P O E$ polymorphisms were shown in Additional file 1: Table S7. The distribution of $A P O E$ genotypes in normal lipid phenotype and/or isolated low HDL-C phenotype fullfilled Hardy-Weinberg equilibrium $\left(x^{2}=6.18\right.$, $p=0.186 ; \chi^{2}=5.20, p=0.267 ; \chi^{2}=9.37, p=0.052$; Additional file 1: Table S7), suggesting that the sampling bias problem in this study was avoided. Multinomial logistic regression analysis showed that there were no association between $A P O E$ allele carriers and the two miRNA candidates as well as their original $\mathrm{Ct}$ ratio (Additional file 1: Table S8).

\section{Discussion}

Several studies has been attempted to explore a set of circulating miRNA candidates potential for clinical utilities on diagnosis and prevention of cardiovascular diseases [10]. Although the differentially expressed circulating miRNAs in cardiovascular diseases could be found easily, most of circulating miRNA candidates could lose statistical significance once adjusted for various risk factors such as age, gender, smoking, lipid indexes, hypertension and metabolic diseases [12-15]. It is necessary to investigate cardiovascular disease-derived miRNAs in isolated risk phenotype prior to onset of cardiovascular diseases. Herein, in this study, we performed the baseline investigation of CVD-derived circulating miR-221/222 mature transcripts as well as APOE genotyping in asymptomatic subjects to enhance our understandings on their molecular pathological features in specific risk phenotype. We found that subjects with isolated low HDL-C phenotype exhibit increased levels of miR222-3p and decreased levels of miR-221-3p in plasma. Female subjects with either isolated low HDL-C phenotype or normal lipid phenotype exhibited higher miR-221-3p levels than male subjects. Circulating miR-222-3p levels are robustly associated with isolated low HDL-C phenotype. Moreover, the inverted pattern of circulating miR-221-3p and miR-222-3p in isolated low HDL-C phenotype provide us an opportunity to apply the original $\mathrm{Ct}$ ratio to annotating molecular pathology of isolated low HDL-C phenotype.

MIR-221/222 has been reported to play important roles in various physiological and pathological processes in
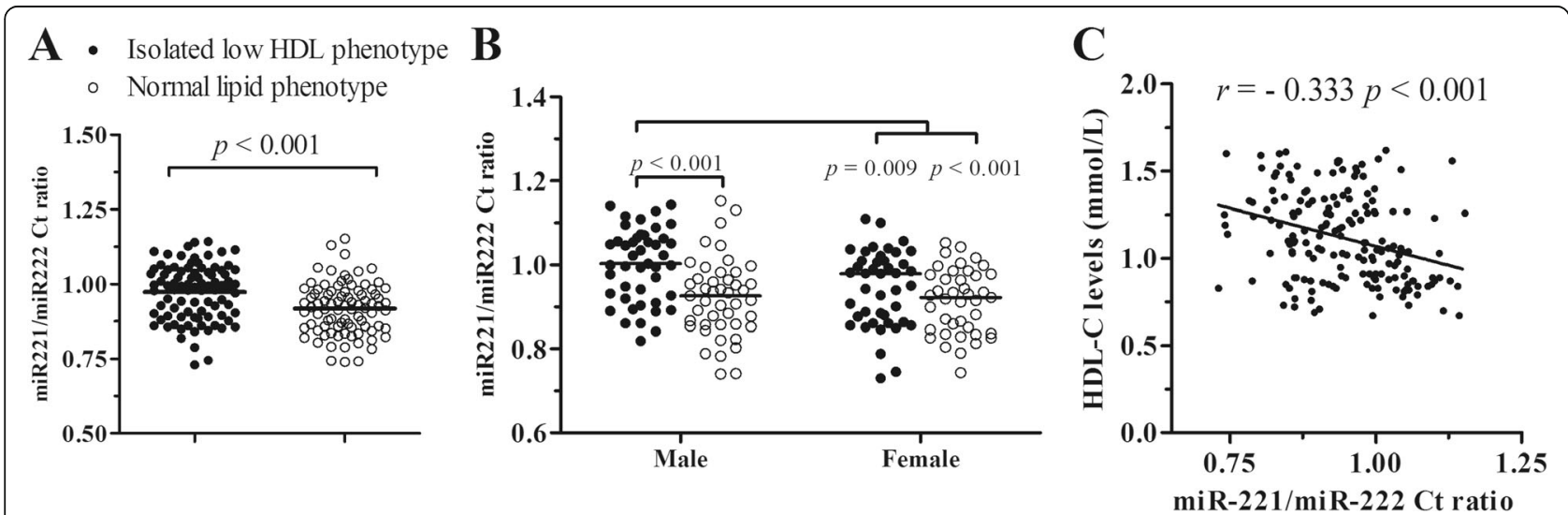

Fig. 3 The ratio of miR-221-3p and miR-222-3p and isolated low HDL-C phenotype. a The original Ct ratio of circulating miR-221-3p to miR-222-3p are significantly elevated in isolated low HDL-C phenotype. b Compared with the corresponding normal lipid phenotype, male subjects with isolated low HDL phenotype exhibit the increased miR-221-3p/222-3p ratio, while the female isolated low HDL-C phenotype have a increased trendence. $\mathbf{c}$ The original Ct ratio of miR-221-3p to miR-222-3p are inversely correlated with serum HDL-C levels in asymptomatic subjects $(n=174)$. The $p$ value is adjusted using the Benjamini-Hochberg $(\mathrm{BH})$ method in multiple comparisons 
Table 3 The AUCs' performance for distinguishing subjects with isolated low HDL-C phenotype in gender-based analysis

\begin{tabular}{|c|c|c|c|c|c|}
\hline Models & AUC $(95 \% \mathrm{Cl})$ & $p$ value & Cut-off value & Specificity & Sensitivity \\
\hline \multicolumn{6}{|l|}{ Male subjects } \\
\hline miR-222-3p & $0.671(0.566,0.730)$ & 0.002 & 0.474 & 0.667 & 0.622 \\
\hline miR-221-3p/miR-222-3p ratio & $0.731(0.626,0.836)$ & $<0.001$ & 0.564 & 0.800 & 0.622 \\
\hline Clinical model & $0.759(0.659,0.859)$ & $<0.001$ & 0.630 & 0.867 & 0.556 \\
\hline Clinical model + miR-222-3p $p^{a}$ & $0.813(0.721,0.904)$ & $<0.001$ & 0.522 & 0.800 & 0.778 \\
\hline Clinical model + miR-221-3p/miR-222-3p ratio ${ }^{a}$ & $0.851(0.770,0.933)^{c}$ & $<0.001$ & 0.430 & 0.756 & 0.889 \\
\hline \multicolumn{6}{|l|}{ Female subjects } \\
\hline miR-222-3p & $0.623(0.501,0.745)$ & 0.026 & 0.600 & 0.878 & 0.417 \\
\hline miR-221-3p/miR-222-3p ratio & $0.624(0.503,0.745)$ & 0.025 & 0.566 & 0.780 & 0.512 \\
\hline Clinical model & $0.808(0.714,0.901)$ & $<0.001$ & 0.548 & 0.854 & 0.674 \\
\hline Clinical model + miR-222-3p & $0.847(0.761,0.934)$ & $<0.001$ & 0.421 & 0.780 & 0.837 \\
\hline Clinical model + miR-221-3p/miR-222-3p ratio ${ }^{b}$ & $0.841(0.756,0.926)$ & $<0.001$ & 0.556 & 0.853 & 0.721 \\
\hline
\end{tabular}

AUC area under the curve. Best threshold of Cut-off value were estimated using youden method

$a, b$ The AUC, sensitivities and specificities of the combination model of either miR-222-3p or miR-221-3p/miR-222-3p ratio and clinical indexes were compared with that of the predictive clinical model for male and female subjects, respectively ${ }^{c} p<0.05$

cardiovascular system [32], suggesting that miR-221/222 may be a potential cardiovascular biomarker and a new therapeutic target in cardiovascular diseases. The levels of miR-221/222 mature transcripts in different circulating cell-free conditions including plasma, serum or microvesicles were investigated in cardiovascular risk factors such as aging [33], endurance training [34, 35], obesity [30, 31, 36, 37], T2DM [38], stable acute coronary syndrome [6], nonST-segment elevation myocardial infarction (NSTEMI) [39], and atherosclerosis [40, 41]. Our findings revealed the inverted pattern of decreased circulating miR-221-3p versus increased miR-222-3p levels in isolated low HDL-C phenotype in comparison with normal lipid phenotype, which is similar to the pattern of miR-221/222-3p in morbidly obese patients [30]. Notably, circulating miR-222-3p levels were robustly associated with isolated low HDL-C phenotype, whereas miR-221-3p exhibited the stronger association with gender factor than miR-222-3p. miR-221-3p and/or miR-222-3p have been shown to be associated with body mass index [31], weights [36] and endurance training [42]. It is well known that the reduced cardiovascular risk should be beneficial from raising HDL-C level, modest weight loss and aerobic exercise. These features of miR-221-3p/miR-222-3p in cell-free conditions support their roles in cardiovascular system and could become a promosing target to cardiovascular health management.

There are various types of miRNA carriers including lipoproteins, exosome and microparticles in circulation system [43]. A set of HDL-transported miRNAs such as miR-222 were found to be varied with HDL subfraction from subjects with familial hypercholesterolemia [26]. With the increase of HDL-C levels, more and more miR-222 should be transported into circulation through HDL particle. However, it is hardly ascertained whether its integrated levels in plasma sample including HDL, LDL, exosome as well as other carriers exhibt the same pattern as that in HDL particle alone. In this study, plasma miR-221-3p, rather than miR-222-3p, were increased with HDL-C levels, and this increases should be attributed to the difference between female and male. Nevertheless, subjects with higher HDL-C levels exhibited lower plasma miR-222-3p levels. The pattern of plasma miR-221-3p and miR-222-3p in isolated low HDL-C phenotype is contradictory to the mechanism on coordinated transcription of miR-221/222 through estrogen receptor alpha (ER $\alpha)$-mediated inhibition in cells [24]. In addition, ApoE isoforms are another transcriptional repressor for miR-221/222 [25]. Unfortunately, ApoE allele carriers were not associated with this pattern at the genetic level. Although its mechanism remains unknown, the inverted pattern of miR-221-3p and miR-222-3p as well as the female-specific increases in miR-221-3p in cell-free conditions were also reported in patients with morbid obesity [30] or metabolic syndrome [31]. It is well known that subjects with morbid obesity or metabolic syndrome usually represent low HDL-C levels and high TG levels. Taken together these evidences, the specific pattern of extracellular miR-221/ 222-3p may be distinctive for increased cardiovascular risk.

There have always been several key issues such as reference selection for circulating miRNA quantification and the accuracy of single-miRNA assay in the translation of biomarkers into clinical practice. It is widely recommended to use one or a panel of invariant endogenous normalizers and/or exogenous synthetic small RNA for miRNA qPCR quantification, and relative expression levels are reported as $-\Delta \mathrm{Ct}$ or $-\Delta \Delta \mathrm{Ct}$ and the log2-transformed value [44]. In this study, the inverted signature of 
plasma miR-221-3p and miR-222-3p in isolated low HDL-C phenotype made us achieve the substitution of the original Ct ratio of miR-221-3p to miR-222-3p for classic qPCR quantification method to provide more comprehensive and accuracy information as a mirror of miR-221/222 gene at circulating plasma level for predicting isolated low HDL-C phenotype, especially in distinguishing male subjects with isolated low HDL-C phenotype type from normal lipid phenotype. Moreover, its usage overcame the uncertainty of reference selection for circulating plasma miRNAs. The method using the ratio of miR-221/222-3p in plasma sample should be at least suitable for isolated low HDL-C phenotype and morbid obesity although it could be affected by other potentially pathological conditions.

\section{Conclusions}

The relationship between isolated low HDL-C phenotype/low HDL-C level and cardiovascular diseases has been explored in the context of Epidemiology, genetics and HDL dysfunctional. We explored the molecular pathological feature of isolated low HDL-C phenotype via profiling CVD-derived circulating miRNA candidates and found the robust association between miR-222-3p and isolated low HDL-C phenotype, and the inverted pattern of miR-221-3p/222-3p and female-specific elevation of miR-221-3p. The use of the original miR-221-3p and 222-3p Ct values exclusively exhibits the advantage over classic miRNA quantification analysis for clinical translation. Given the dominant role of miR-221/222 in vascular biology and atherosclerotic disease, the specific pattern for isolated low HDL-C phenotype combined with serum HDL-C testing may be useful in healthy management of isolated CVD risk phenotype. In the following study, we would explore whether both the inverted pattern and the original $\mathrm{Ct}$ ratio of miR-221/ 222 in cell-free circulating status are suitable for early diagnosis and prevention of major adverse cardiovascular event in isolated low HDL-C phenotype risk population and investigate their patterns in distinct isolated cardiovascular risk phenotypes including isolated high LDL-C and TG phenotypes.

\section{Additional file}

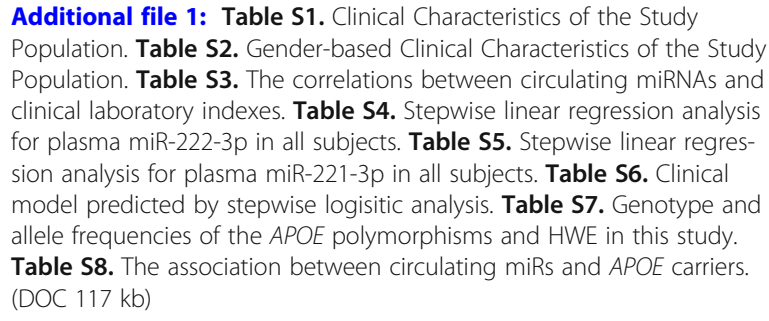

\section{Abbreviations}

ACS: Acute coronary syndrome; ALT: Alanine aminotransferase; AST: Aspartate aminotransferase; CVD: Cardiovascular disease; FG: Fasting glucose; GGT: Gammaglutamyl transferase; HDL: High-density lipoprotein; HGB: Hemoglobin; HWE: Hardy-Weinberg equilibrium; ILHP: Isolated low HDL-C phenotype; LDL: Low-density lipoprotein; LYM: Lymphocyte; miRNAs: MicroRNAs; NEU: Neutrophill; NSTEMI: Non-ST-segment elevation myocardial infarction; PLT: Platelets; RBC: Red blood cell; TC: Total cholesterol; TG: Triglyceride; UAP: Unstable angina pectoris; WBC: White blood cell

\section{Acknowledgements}

The authors gratefully thank Dr. David Deng for minor revision.

\section{Funding}

This study was supported by grant from Key Laboratory Development Program sponsored by Tangshan Science and Technology Bureau $(17140210 \mathrm{~A})$. The sponsor has no role in study design, data collection and analysis, results interpretation or in preparation, review and approval of the manuscript.

\section{Availability of data and materials}

The datasets used during the study are available from the corresponding author on reasonable request and miRNA microarray dataset can be retrieved from NCBI's gene expression omnibus website (http://www.ncbi.nlm.nih.gov/ geo/, accession number: GSE94605)

\section{Authors' contributions}

WT, YTZ and ZJ conceived and designed the study. MDL, JRL and LS performed the experiments. MDL, JRL, YTZ had repeatedly checked all of the data and takes the responsibility for the integrity of the data and the accuracy of data analysis. YTZ and WT drafted the final manuscript. All authors approved the final manuscript.

\section{Ethics approval and consent to participate}

The protocol on participant enrollment and plasma sample collection was approved by the Ethics Committee of Tangshan Gongren Hospital (GRYY2012-20). Each study participant provided written informed consent.

\section{Competing interests}

The authors declare that they have no competing interests.

\section{Publisher's Note}

Springer Nature remains neutral with regard to jurisdictional claims in published maps and institutional affiliations.

Received: 21 March 2018 Accepted: 3 August 2018

Published online: 16 August 2018

\section{References}

1. Alvarez-Garcia I, Miska EA. MicroRNA functions in animal development and human disease. Development. 2005;132:4653-62.

2. Wang J, Chen J, Sen S. MicroRNA as biomarkers and diagnostics. J Cell Physiol. 2016;231:25-30.

3. Canfran-Duque A, Lin CS, Goedeke L, Suarez Y, Fernandez-Hernando C. Micro-RNAs and high-density lipoprotein metabolism. Arterioscler Thromb Vasc Biol. 2016;36:1076-84

4. van Rooij E. Introduction to the series on MicroRNAs in the cardiovascular system. Circ Res. 2012;110:481-2.

5. Corsten MF, Dennert $R$, Jochems $S$, Kuznetsova $T$, Devaux $Y$, Hofstra L, Wagner DR, Staessen JA, Heymans S, Schroen B. Circulating MicroRNA-208b and MicroRNA-499 reflect myocardial damage in cardiovascular disease. Circ Cardiovasc Genet. 2010;3:499-506.

6. Jansen F, Yang X, Proebsting S, Hoelscher M, Przybilla D, Baumann K, Schmitz T, Dolf A, Endl E, Franklin BS, Sinning JM, Vasa-Nicotera M, Nickenig G, Werner N. MicroRNA expression in circulating microvesicles predicts cardiovascular events in patients with coronary artery disease. J Am Heart Assoc. 2014;3:e001249.

7. Kuwabara Y, Ono K, Horie T, Nishi H, Nagao K, Kinoshita M, Watanabe S, Baba O, Kojima Y, Shizuta S, Imai M, Tamura T, Kita T, Kimura T. Increased microRNA1 and microRNA-133a levels in serum of patients with cardiovascular disease indicate myocardial damage. Circ Cardiovasc Genet. 2011;4:446-54. 
8. Wang F, Long G, Zhao C, Li H, Chaugai S, Wang Y, Chen C, Wang DW. Plasma microRNA-133a is a new marker for both acute myocardial infarction and underlying coronary artery stenosis. J Transl Med. 2013; 11:222.

9. Contu R, Latronico MVG, Condorelli G. Circulating MicroRNAs as potential biomarkers of coronary artery disease a promise to be fulfilled? Circ Res. 2010;107:573-4

10. Navickas R, Gal D, Laucevičius A, Taparauskaitẻ A, Zdanytè M, Holvoet P. Identifying circulating microRNAs as biomarkers of cardiovascular disease: a systematic review. Cardiovasc Res. 2016;111:322-37.

11. Liu J, Liu Y, Sun Y-N, Li S, Liu X-Q, Li J, Li C-M, Tian W, Zhou Y-T, Shang X-M. miR-28-5p involved in LXR-ABCA1 pathway is increased in the plasma of unstable angina patients. Heart Lung Circ. 2015;24:724-30.

12. Shuji Sawada TA, Takahashi M, Sakurai R, Shinkai S, Ushida T, Fujiwara Y, Suzuki K. Effect of Aging and Sex on Circulating MicroRNAs in Humans. Adv Aging Res. 2014;3:152-9.

13. Takahashi K, Yokota S-i, Tatsumi N, Fukami T, Yokoi T, Nakajima M. Cigarette smoking substantially alters plasma microRNA profiles in healthy subjects. Toxicol Appl Pharmacol. 2013;272:154-60.

14. Romaine SP, Charchar FJ, Samani NJ, Tomaszewski M. Circulating microRNAs and hypertension--from new insights into blood pressure regulation to biomarkers of cardiovascular risk. Curr Opin Pharmacol. 2016;27:1-7.

15. Párrizas $M$, Novials A. Circulating microRNAs as biomarkers for metabolic disease. Best Pract Res Clin Endocrinol Metab. 2016;30:591-601.

16. Goldbourt U, Yaari S, Medalie JH. Isolated low HDL cholesterol as a risk factor for coronary heart disease mortality: a 21-year follow-up of 8000 men. Arterioscler Thromb Vasc Biol. 1997;17:107-13.

17. Huxley RR, Barzi F, Lam TH, Czernichow S, Fang X, Welborn T, Shaw J, Ueshima H, Zimmet P, Jee SH, Patel JV, Caterson I, Perkovic V, Woodward M. Isolated low levels of high-density lipoprotein cholesterol are associated with an increased risk of coronary heart disease - an individual participant data meta-analysis of 23 studies in the Asia-Pacific region. Circulation. 2011; 124:2056-64.

18. Weissglas-Volkov D, Pajukanta P. Genetic causes of high and low serum HDL-cholesterol. J Lipid Res. 2010;51:2032-57.

19. Bisoendial RJ, Hovingh GK, Levels JH, Lerch PG, Andresen I, Hayden MR, Kastelein JJ, Stroes ES. Restoration of endothelial function by increasing high-density lipoprotein in subjects with isolated low high-density lipoprotein. Circulation. 2003;107:2944-8.

20. Holven KB, Retterstøl K, Ueland T, Ulven SM, Nenseter MS, Sandvik M, Narverud I, Berge KE, Ose L, Aukrust P, Halvorsen B. Subjects with low plasma HDL cholesterol levels are characterized by an inflammatory and oxidative phenotype. PLoS One. 2013;8:e78241.

21. Yetukuri L, Soderlund S, Koivuniemi A, Seppanen-Laakso T, Niemela PS, Hyvonen M, Taskinen MR, Vattulainen I, Jauhiainen M, Oresic M. Composition and lipid spatial distribution of HDL particles in subjects with low and high HDL-cholesterol. J Lipid Res. 2010;51:2341-51.

22. Nakanishi S, Vikstedt R, Soderlund S, Lee-Rueckert M, Hiukka A, Ehnholm C, Muilu M, Metso J, Naukkarinen J, Palotie L, Kovanen PT, Jauhiainen M, Taskinen MR. Serum, but not monocyte macrophage foam cells derived from low HDL-C subjects, displays reduced cholesterol efflux capacity. J Lipid Res. 2009;50:183-92.

23. Vazzana N, Ganci A, Cefalù AB, Lattanzio S, Noto D, Santoro N, Saggini R, Puccetti L, Averna M, Davi G. Enhanced lipid peroxidation and platelet activation as potential contributors to increased cardiovascular risk in the low-HDL phenotype. J Am Heart Assoc. 2013;2:e000063.

24. Di Leva G, Gasparini P, Piovan C, Ngankeu A, Garofalo M, Taccioli C, lorio MV, Li M, Volinia S, Alder H, Nakamura T, Nuovo G, Liu Y, Nephew KP, Croce CM. MicroRNA cluster 221-222 and estrogen receptor a interactions in breast Cancer. J Natl Cancer Inst. 2010;102:706-21.

25. Kothapalli D, Castagnino P, Rader DJ, Phillips MC, Lund-Katz S, Assoian RK. Apolipoprotein E-mediated cell cycle arrest linked to p27 and the Cox2dependent repression of miR221/222. Atherosclerosis. 2013;227:65-71.

26. Vickers KC, Palmisano BT, Shoucri BM, Shamburek RD, Remaley AT. MicroRNAs are transported in plasma and delivered to recipient cells by high-density lipoproteins. Nat Cell Biol. 2011;13:423-33.

27. Mestdagh P, Van Vlierberghe P, De Weer A, Muth D, Westermann F, Speleman $F$, Vandesompele J. A novel and universal method for microRNA RT-qPCR data normalization. Genome Biol. 2009;10:R64.

28. Zhang M-d, Gu W, Qiao S-b, Zhu E-j, Zhao Q-m, Lv S-z. Apolipoprotein E gene polymorphism and risk for coronary heart disease in the Chinese population: a meta-analysis of 61 studies including 6634 cases and 6393 controls. PLoS One. 2014;9:e95463.

29. Harper $\mathrm{CR}$, Jacobson TA. Treating isolated low high-density lipoprotein cholesterol: prescient or premature? Am J Cardiol. 2000;85:484-6.

30. Ortega FJ, Mercader JM, Catalan V, Moreno-Navarrete JM, Pueyo N, Sabater M, Gomez-Ambrosi J, Anglada R, Fernandez-Formoso JA, Ricart W, Fruhbeck G, Fernandez-Real JM. Targeting the circulating microRNA signature of obesity. Clin Chem. 2013:59:781-92.

31. Wang YT, Tsai PC, Liao YC, Hsu CY, Juo SH. Circulating microRNAs have a sex-specific association with metabolic syndrome. J Biomed Sci. 2013;20:72.

32. Chistiakov DA, Sobenin IA, Orekhov AN, Bobryshev YV. Human miR-221/222 in physiological and atherosclerotic vascular remodeling. Biomed Res Int. 2015;2015:354517.

33. Zhang H, Yang H, Zhang C, Jing Y, Wang C, Liu C, Zhang R, Wang J, Zhang J. Zen K, Zhang C, Li D. Investigation of MicroRNA expression in human serum during the aging process. J Gerontol A Biol Sci Med Sci. 2015;70:102-9.

34. Baggish AL, Hale A, Weiner RB, Lewis GD, Systrom D, Wang F, Wang TJ, Chan SY. Dynamic regulation of circulating microRNA during acute exhaustive exercise and sustained aerobic exercise training. J Physiol. 2011;589:3983-94.

35. Nielsen S, Akerstrom T, Rinnov A, Yfanti C, Scheele C, Pedersen BK, Laye MJ. The miRNA plasma signature in response to acute aerobic exercise and endurance training. PLoS One. 2014;9:e87308.

36. Prats-Puig A, Ortega FJ, Mercader JM, Moreno-Navarrete JM, Moreno M, Bonet N, Ricart W, Lopez-Bermejo A, Fernandez-Real JM. Changes in circulating microRNAs are associated with childhood obesity. J Clin Endocrinol Metab. 2013;98:E1655-60

37. Parr EB, Camera DM, Burke LM, Phillips SM, Coffey VG, Hawley JA. Circulating MicroRNA responses between 'High' and 'Low' responders to a 16-Wk diet and exercise weight loss intervention. PLoS One. 2016;11:e0152545.

38. Ortega FJ, Mercader JM, Moreno-Navarrete JM, Rovira O, Guerra E, Esteve E, Xifra G, Martinez C, Ricart W, Rieusset J, Rome S, Karczewska-Kupczewska M, Straczkowski M, Fernandez-Real JM. Profiling of circulating microRNAs reveals common microRNAs linked to type 2 diabetes that change with insulin sensitization. Diabetes Care. 2014;37:1375-83.

39. Ward JA, Esa N, Pidikiti R, Freedman JE, Keaney JF, Tanriverdi K, Vitseva O, Ambros V, Lee R, McManus DD. Circulating cell and plasma microRNA profiles differ between non-ST-segment and ST-segment-elevation myocardial infarction. Fam Med Med Sci Res. 2013;2:108.

40. Zhang X, Shao S, Geng H, Yu Y, Wang C, Liu Z, Yu C, Jiang X, Deng Y, Gao L, Zhao J. Expression profiles of six circulating microRNAs critical to atherosclerosis in patients with subclinical hypothyroidism: a clinical study. J Clin Endocrinol Metab. 2014;99:E766-74.

41. He XM, Zheng YQ, Liu SZ, Liu Y, He YZ, Zhou XY. Altered plasma MicroRNAs as novel biomarkers for arteriosclerosis obliterans. J Atheroscler Thromb. 2016:23:196-206.

42. Wardle SL, Bailey ME, Kilikevicius A, Malkova D, Wilson RH, Venckunas T, Moran CN. Plasma microRNA levels differ between endurance and strength athletes. PLoS One. 2015;10:e0122107.

43. Boon RA, Vickers KC. Intercellular transport of MicroRNAs. Arterioscler Thromb Vasc Biol. 2013;33:186-92

44. Marabita F, de Candia P, Torri A, Tegner J, Abrignani S, Rossi RL. Normalization of circulating microRNA expression data obtained by quantitative real-time RTPCR. Brief Bioinform. 2016;17:204-12.

Ready to submit your research? Choose BMC and benefit from:

- fast, convenient online submission

- thorough peer review by experienced researchers in your field

- rapid publication on acceptance

- support for research data, including large and complex data types

- gold Open Access which fosters wider collaboration and increased citations

- maximum visibility for your research: over $100 \mathrm{M}$ website views per year

At BMC, research is always in progress.

Learn more biomedcentral.com/submissions 